\title{
STAATEN UND LANDSCHAFTEN IN INDIEN
}

\author{
KARL, KRÜGER
}

Mit einer Karte

Die Einzelstaaten der «Union von Indien» (Bharat $=$ 《Heimat») entsprechen nicht natürlichen Landschaften. Hier soll deshalb versucht werden, eine «natürliche » Gruppierung zu erreichen.

Die in Quadratkilometern (1 Quadratmeile $=2,58944 \mathrm{~km}^{2}$ ) errechneten Flächen stehen noch nicht in ihren Hunderten, $z$. T. nicht in den Tausenden fest, zumal neue indische Veröffentlichungen ${ }^{1}$ zwar die Gesamtfläche mit 1220099 Quadratmeilen angeben, aber in den gleichen Aufstellungen melirere Einzelstaaten mit ihren Flächen von 1941 bringen, so daß sich eine Differenz von über 100000 Quadratmeilen ergibt. Unter Benutzung des India and Pakistan Year-Book 1949 (Bombay 1950) und anderer Quellen. konnten vorläufige Werte in Quadratkilometern errechnet werden, die einigermaßen befriedigen. Die Bevölkerungszahlen wurden gemäß der Schätzung vom März 1950 eingesetzt. Bisher wurde die Zahl der Flüchtlinge aus Pakistan mit 6-7 Millionen angegeben, neuerdings jedoch mit 9 Millionen. Infolgedessen ist anzunehmen, daß unter Berücksichtigung des natürlichen Bevölkerungszuwachses die Gesamtbevölkerung der Republik Indien jetzt schon 350 Millionen erreicht. Für die große Volks- und Wirtschaftszählung vom März 1951 - den alle zehn Jahre vorgenommenen Census - wurden 350,67 Millionen verausherechnet.

Die Zentrairegieruug in Delhi erstrebt eine Neueinteilung der Union vornehmlich nach sprachlichen Gesichtspunkten, so daß sich linguistic provinces ergeben könnten. Die Durchsetzung des Hindi als Unionssprache stößt auf Schwierigkeiten; Hindi, mit Sanskrit-Silbenschrift $=$ Devanagari geschrieben, wird möglicherweise ab 1964 sogar Englisch als Amtssprache ausschalten. Daneben werden die Gebictssprachen anerkannt; es ist bezeichnend, daß die Hauptradiostationen der Republik Indien je 243 Stunden außer Englisch und Hindi auch Tamil und Telugu (Südindien), Bengali (für das untere Gangesland, aber auch Ost-Pakistan), Gudscherati und Marathi (Bombay-Hinterland), Kannada (= Kanaresisch; Maisor = Mysore, Süd-Bombey), Assamisch, Pandschabi und mit geringerer Stundenzahl Kaschmiri und Dogri sowie Gurkhali senden. Urdu (Hindustani in besonderem Sinne), die altc Basarsprache aus der Zeit der Moghulkaiser und spätere Befehlssprache des Militärs, wird im Auslanddienst gesendet. Urdu ist auch in der Republik Indien (z. B. U.P., Bhopal usw.) weit verbreitet, sonst als Handelssprache bekannt (In Pakistan ist Urdu Amtssprache [Englisch ist zugelassen]).

Sowohl in der Union von Indien, als auch in Pakistan, ist die englische Schreibweise üblich. Neuerdings wird auch amtlich eine reformierte Orthographie für manche Namen angewandt: Kanpur statt Cawnpore, Ambala statt Umballa, Bareli statt Bareilly usw., in Pakistan Panjab statt Punjab. Im Folgenden seien einige Aussprachewinke gegeben (in Klammern englische bezw. amtliche Schreibweise): Dschammu (Jammu), Bihar (Behar, auch Bihar), Schimla (Simla), Radschast'han (Rajasthan), Katsch (Kutch, auch Katch), Radschkot (Rajkot), Haidarabad (Hyderabad, auch Hydarabad), Maisor (Mysore), Kurg (Coorg, auch Kurg), Trawankor-Kotschin (Tratancore-Cochin, nicht 'Trawankur), Katsch-Bihar (Cutch-Behar), Tschittagong (Chittagong), Karàtschi (Karachi), Chairpur (Khairpur), Charan (Kharan), Tschitral (Chitral). Man muß zwischen dem (arabischen) Kh des Urdu, das ein Rachen-Ch ist, und dem $\mathrm{Kh}$ des Sanskrit in Hindi usw. unterscheiden, das als $\mathrm{K}$ 'h aspiriert gesprochen wird (Dekkhan). Kwetta ist richtiger als Ketta (Quetta).

DIE S'IAA'TEN DER UNION VON INDIEN (Hauptstädte in Klammern)

Himalaja-Gibiet im zuestlichen Norden

Jammu und Kaschmir (B) (Srinagar).

liiinfstromland
Fläche $1000 \mathrm{~km}=$ Bevölkerung 1950 Millionen

$\begin{array}{rr}21.3,1 & 4,37 \\ 27,5 & 1,08 \\ 95,8 & 12,61 \\ 26,1 & 3,32 \\ 1,2 & 0,13\end{array}$

1 India Record, Bd. 2, Nr. 11; 16. März 1950; High Commissioner for India, London. S. 8. About India; Ministry of Information and Broadcasting. Delhi, August 1949, S. 45. 


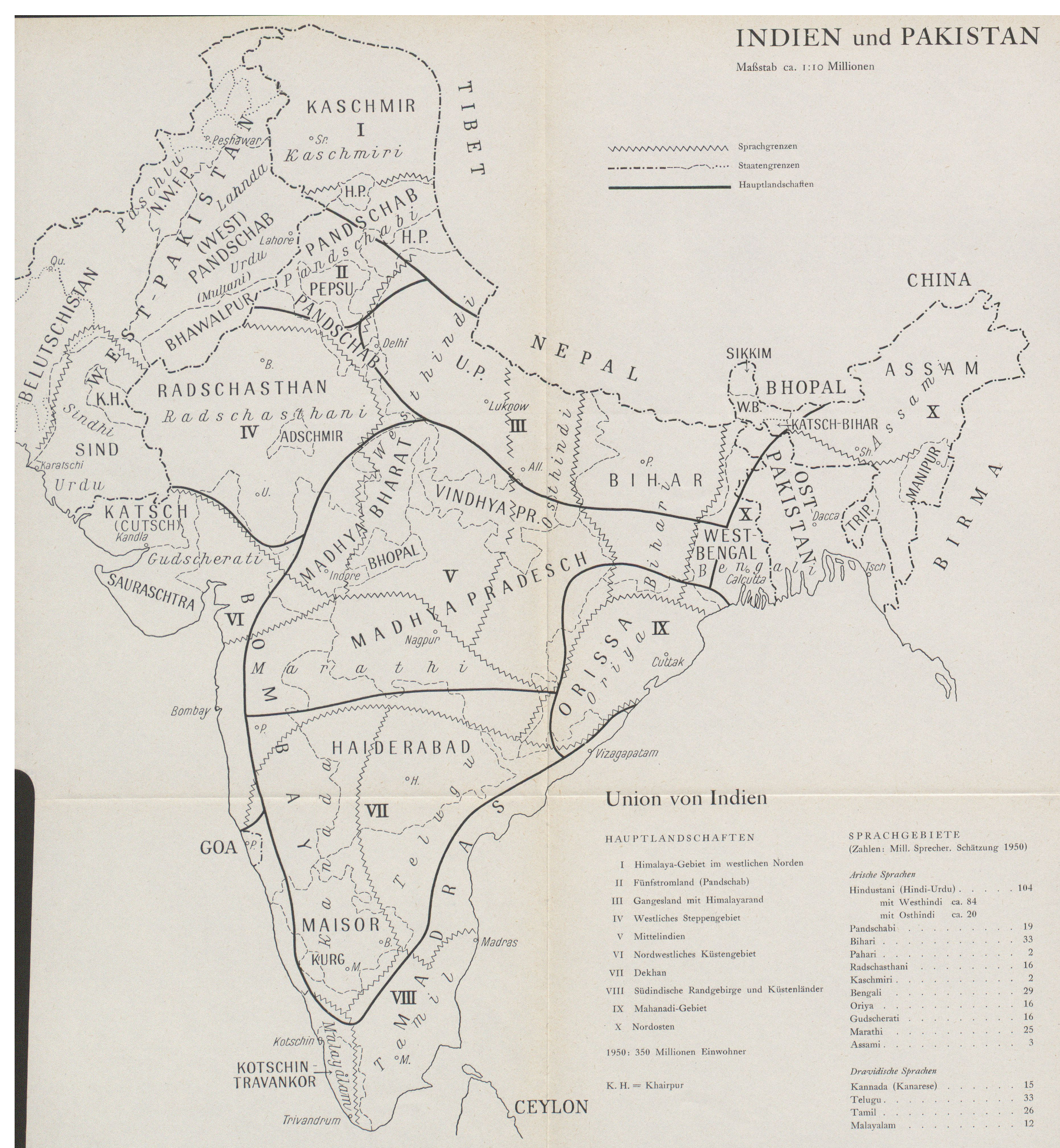


U.P. (Uttar Pradesh) (früh. Un. Prov.) (A) (Lucknow)

Delhi $(C, x)$ (Hauptstadt der Union) . . . . .

Bihar (A) (Patna).

Sikkim (Protektorat) (Gangtok) . . . . . .

Irestliches Steppengebiet (Thar\%, Rudschputana)

Rajasthan (B) (Jaipur) . . . .

Ajmer (-Merwara) (C, x) (Ajmer) . . . . . .

Mittclindien

Madhya Bharat (B) (Gwalior) . . . . . .

Madhya Pradesh (früh. Central Prov.) (A) (Nagpur)

Bhopal (C) (Bhopal) . . . . . . . . . .

Vindhya Pradesh (B zeitweise C).(Rewa). . .

Nordivestliches Kiistengebict, mit Gudscherat

Kutch (C) (Bhuj)

Saurashtra (früh. Katiawar-Staiten) (B) (Radjkot)

Bombay (A) (Bombay).

Dekkhan

Hyderabad (B) (Hyderabad)

Mysore (B) (Mysore)

Coorg $(\mathrm{C}, \mathrm{x})$ (Mercara) .

Sïdindische Randgebirge und Küstenländer

Travancore-Cochin (B) (Trivandrum) . . . . . 23,

Madras (A) (Madras) . . . . . . . . . . .

Malanadi-Gebict

Orissa (A) (im Bau nördlich Bhubaneshvar). . . 155

Nordosten: Deltaland, Brahmaputragebict

(West)Bengal (A) Calcutta) (mit Cutch Behar). . 75,3

Assam (A) (Shillong) . . . . . . . .

Tripura (C) (Agartila) . . . . . . . . . .

Manipur (C) (Manipur).

Andamanc" und Nikobaren

(C, x) (Port Blair)

(Aus der amtlichen Angabe der Gesamtfiliche von 1220099

Quadrat-Meilen [ohne Sikkim?] errechnet
275,1

1,3

180,7

7,1

61,62

1,51

39,42

0,13

$362,5 \quad 14,69$

$6,2 \quad 0,73$

120,1

$3+1,1 \quad 20,92$

$26,1 \quad 0,85$

$63,7 \quad 3,88$

$21,9 \quad 0,55$

$5 \overline{5}, 2 \quad 3,96$

$296,7 \quad 32,68$

$213,2 \quad 17,69$

$76,3 \quad 8,08$

$4,1 \quad 0,17$

$3,7 \quad 5,58$

$5+, 29$

14,41

$75,3 \quad 24,32$

$130,3 \quad 8,51$

$10,7 \quad 0,58$

$22,3 \quad 0,54$

$\begin{array}{cc}8,0 & 0,30 \\ 3166,0 & 347,80\end{array}$

3159,4

(A): frühere Governor's Provinces, heute = A-Statten.

(B): frühere Fürstenstaaten = B-Staaten; mit Ausnahme von Mysore, Hyderabad, Jammu/Kaschmir zu Unionen zusammengeschlossen.

(C): zentral verwaltete Gebiete $=$ C-Staaten.

$\mathrm{x}$ : frühere Chief Commissioner's Provinces.

Pradesh $=$ Provinz, Uttar $=$ Norden, Madhya $=$ Mitte. Himachal (Hima-tschal) ist eine historische Bezeichnung für Himalaja.

Das Bulletin der indischen Mission Bonn, Nr. 2, Oktober 1950 brachte inzwischen eine Übersicht der Flächen (und der Bevölkerung) der Union von Indien, die von unseren Angaben mehr oder weniger abweicht. Zunächst fällt auf, daß die Gesamtfliche mit $3050248 \mathrm{~km}^{2}$ angegeben wird, also $100000 \mathrm{~km}^{2}$ weniger als der bisher veröffentlichten Fläche von 1220099 Quadratmeilen (= $3159373 \mathrm{~km}^{2}$ ) entspricht. Ftwa gleichzeitig erschienen in London die $\mathrm{Nr} .43$ und 44 der amtlichen "India News" vom 4. und 11. November, die für West-Bengalen 29066 Quadrat-Meilen (umgerechnet $=75287 \mathrm{~km}^{2}$ ), für Assam 50296 Quadrat-Meilen (= $130239 \mathrm{~km}^{2}$ ) angaben; das Bulletin gibt diese Flächen mit $72665 \mathrm{~km}^{2}$ (W. Bengal) und $125740 \mathrm{~km}^{2}$ (Assam) an. Da unsere Werte denen der "India News" meist entsprechen und zu einem befriedigenden Wert der Gesamtsumme führen, behalten wir unsere Aufstellung bei, weisen aber ausdrïcklich auf die bestehenden und noch zu klärenden Unstimmigkeiten hin. Am größten ist der Unterschied bei Madhya Pradesch $(=\mathrm{C} . \mathrm{P}$. $=$ Central Provinces); clas Bulletin weist $297960 \mathrm{~km}^{2}$ aus, das Yearbook 1949 aber 131686 Quadratmeilen ( $=341000 \mathrm{~km}^{2}$ ); von Abtrennungen ist aber nichts bekannt geworden.

Die Lenkung der A- und B-Staaten obliegt Kabinetten unter je einem Rajpramukh (Radschpramuk'h) $=$ Ministerpräsident bezw. dessen Stellvertreter (Upa- 
oder Uprajpramukh; upa $=$ unter $)$. Die C-Staaten unterstehen Oberkommissaren. Im Mai 1949 beseitigte die Zentralregierung das Kabinett der Vindhya-Union und übernahm die Verantwortung der Regierung. Der kleine Himalajastaat Bilaspur wird im Hinblick auf́ die große nationale Aufgabe des Bhakra/Nangal-Stau- und Kraftwerkes zentral verwaltet. Der Maharadscha von Sikkim unterzeichnete 1950 einen Protektoratsvertrag mit der indischen Zentralregierung. Ein Staatsvertrag mit Bhutan steht noch aus.

Pakistan ist ebenso wie die Republik Indien ein Bundesstaat, blieb aber Dominion unter dem englischen König im Commonwealth; die Union von Indien ist nur angeschlossenes Mitglied des Commonwealth.

Pakistan ist ein islamischer Staat unter cinem Governor-General. Die Provinzen (East Bengal, North West Frontier Province $=N^{\top} W F P$. , Sind, West Panjab stehen unter Governors). Im Staat und in den Provinzen regieren Kabinette unter Führung der Ministerpräsidenten. In Belutschistan ist der Governor-General durch einen Agenten vertreten. Auch Karachi (Karatschi) wird als Hauptstadt zentral v'rwaltet. In den Staaten regieren die Fürsten unter Beratung der Kabinette.

\section{DIF VERIVALTUNGSEINHEITEN VON PAKISTAN? \\ (Hauptstädte in Klammern)}

Fläche $1000 \mathrm{~km}^{2}$ Bevölkerung 1950

Millionen

Ost-Pakistan

East Bengal (Provinz) (Dacca) Divisions: Dacca

West-Pakistan, Unteres Indusland Chittagong, Rajshahi

\begin{tabular}{cc}
140 & 46,7 \\
125 & 4,2 \\
\hline 15 & 1,0 \\
& 0,3
\end{tabular}

Fïnfstromland

Sind (Provinz) (Karachi) . . . . . . . . . 125

Karachi (Hauptstadt, zentral verwaltet)
Khairpur (Fürstenstaat) (Khairpur) . . . . . . . .

0,3

West Panjab (Provinz) (Lahore) . . . . . . . 16119,7

Bahawalpur (Fürstenstaat) (Bahawalpur) . . . . $\quad 45 \quad 1,5$

Nördlicher Gebirgsrand

NWFP. (Provinz) (Peshawar) . . . . . . . . . $\quad 37 \quad 3,2$

Nordivestl. Stammesgebiete (einschl. Amb u. Phulera) $65 \quad 2,5$

Belutschistan und Sïdgrenze Afghanistans

Dir (Dir) $7000 \mathrm{~km}^{2}, 80000 \mathrm{E}$. . . .

Swat (Saidu Sharif) $7000 \mathrm{~km}^{2}$. . . . .

Chitral $10000 \mathrm{~km}^{2}, 80000 \mathrm{E}$.

Baluchistan (zentral verwaltet) (Quetta) . . . . $\quad 141 \quad 0,6$

Fiirstenstaaten in Belutschistan

Kharan (Kharan) . . . . . . . . . . . . . . . . . . . 480,1

Kalat (Kalat) mit Las Bela (Bela) . . . . . . . . $158 \quad 0,4$

West-Pakistan $\quad \frac{795}{935} \quad \ldots \quad \frac{33,5}{80,2}$

2 Vgl. Verfasser: Wirtschaft und Planung in Pakistan. Zeitschrift für Raumforschung, Bielefeld, 1950 , Heft $3 / 5$, Seite 187 .

\section{PAYSAGES ET ÉTATS AUX INDES}

Cet article donne un coup d'œil sur la situation actuelle de la division politique et géographique de l'Inde moderne et du Pakistan.

\section{PAESAGGI E STATI NELLE INDIE}

Questo articolo spiega la situazione attuale della divisione politica e geografica dell'India moderna e del Pakistan. 